\title{
Clinical Reasoning: A 65-year-old woman with subacute hemiballism
}

Kelsey Cacic, MD, Brian Stephens, MD, Francis Cloran, MD, and Anthony Frattalone, MD

Neurology ${ }^{\circledR}$ 2019;92:345-349. doi:10.1212/WNL.0000000000006922

\section{Section 1}

A 65-year-old, right-hand-dominant woman with a history of hypertension, hyperlipidemia, poorly controlled diabetes, and remote breast cancer presented with 1 week of progressive, involuntary left hemibody movements. One month prior, she was a restrained driver in a headon motor vehicle collision with possible brief loss of consciousness. The patient was evaluated at an emergency department and was noted to have musculoskeletal injuries not requiring intervention. No imaging was performed. Since the accident, she described feeling a "mental fog" with slurred speech that has progressively worsened and persistent left shoulder pain.

About 1 week prior to presentation at our facility, she developed nonvoluntary movements starting in her left shoulder, described as "flopping," with gradual progression to incorporate the whole upper limb that spread to the ipsilateral leg and face. These movements became more frequent and increased in amplitude over a week. There was no change in consciousness, loss of bowel/bladder function, or tongue biting during these movements, and no postevent confusion. Due to interference with daily activities, she presented to the emergency department for further evaluation, where a CT head demonstrated a hyperdense lesion in the right basal ganglia with limited surrounding edema.

The patient's vital signs were within normal limits and neurologic examination was notable for mild dysarthria with dyskinetic oromandibular facial movements, intact strength throughout (though limited by left shoulder pain), mild left dysmetria, and left dysdiadochokinesia. At rest, she had intermittent ballistic-choreic movements of her left upper and lower extremities with involvement of the face and tongue, which worsened with intentional maneuvers.

\section{Questions for consideration:}

1. What is your initial differential diagnosis?

2. What laboratory or imaging studies would you perform for further evaluation?

\author{
Correspondence \\ Dr. Cacic \\ Kelsey.a.cacic.mil@mail.mil
}

MORE ONLINE

- Videos

GO TO SECTION 2

From the Department of Neurology (K.C., B.S.), San Antonio Uniformed Services Health Education Consortium; and Departments of Radiology (F.C.) and Neurology (A.F.), San Antonio Military Medical Center, TX.

Go to Neurology.org/N for full disclosures. Funding information and disclosures deemed relevant by the authors, if any, are provided at the end of the article. 


\section{Section 2}

The initial differential of acquired causes of hemichoreahemiballism includes vascular, autoimmune/inflammatory, metabolic, infectious, toxin/drug-induced, and neoplastic or other structural etiologies. ${ }^{1-3}$ In particular, stroke, Wilson disease, thyrotoxicosis, hyperglycemia, and systemic lupus erythematosus have been known to cause this syndrome. ${ }^{1}$ Genetic causes were less likely given age and CT abnormalities. Preliminary laboratory studies demonstrated signs of a urinary tract infection (urinalysis with positive leukocyte esterase and 87 white blood cells) without evidence of systemic infection or inflammation (white blood cells 7.1, erythrocyte sedimentation rate 26) and poorly controlled diabetes (serum glucose 425 , urine trace ketones, $\mathrm{HbAlc} 14)$. Other evidence of infection was not seen in the CSF (negative tuberculosis and bacterial/fungal cultures) or serum (toxoplasma immunoglobulin $\mathrm{G}$ and $\mathrm{M}$ ).
MRI brain with contrast obtained during admission revealed rim-enhancing lesions within the right caudate head and anterior aspect of the right putamen without diffusion restriction but with intralesional hemorrhage (figure). MRI was repeated 2 months later and demonstrated new confluent areas of $\mathrm{T} 1$ hyperintensity involving the right lentiform nucleus sparing the right internal capsule with increasing volume loss of the right caudate head and resolution of $\mathrm{T} 2$ hyperintensity in this region (figure).

\section{Questions for consideration:}

1. What is the differential for rim-enhancing brain lesions on MRI?

2. What is your differential diagnosis for this patient?

3. Is there any historical information on this patient that would be useful?
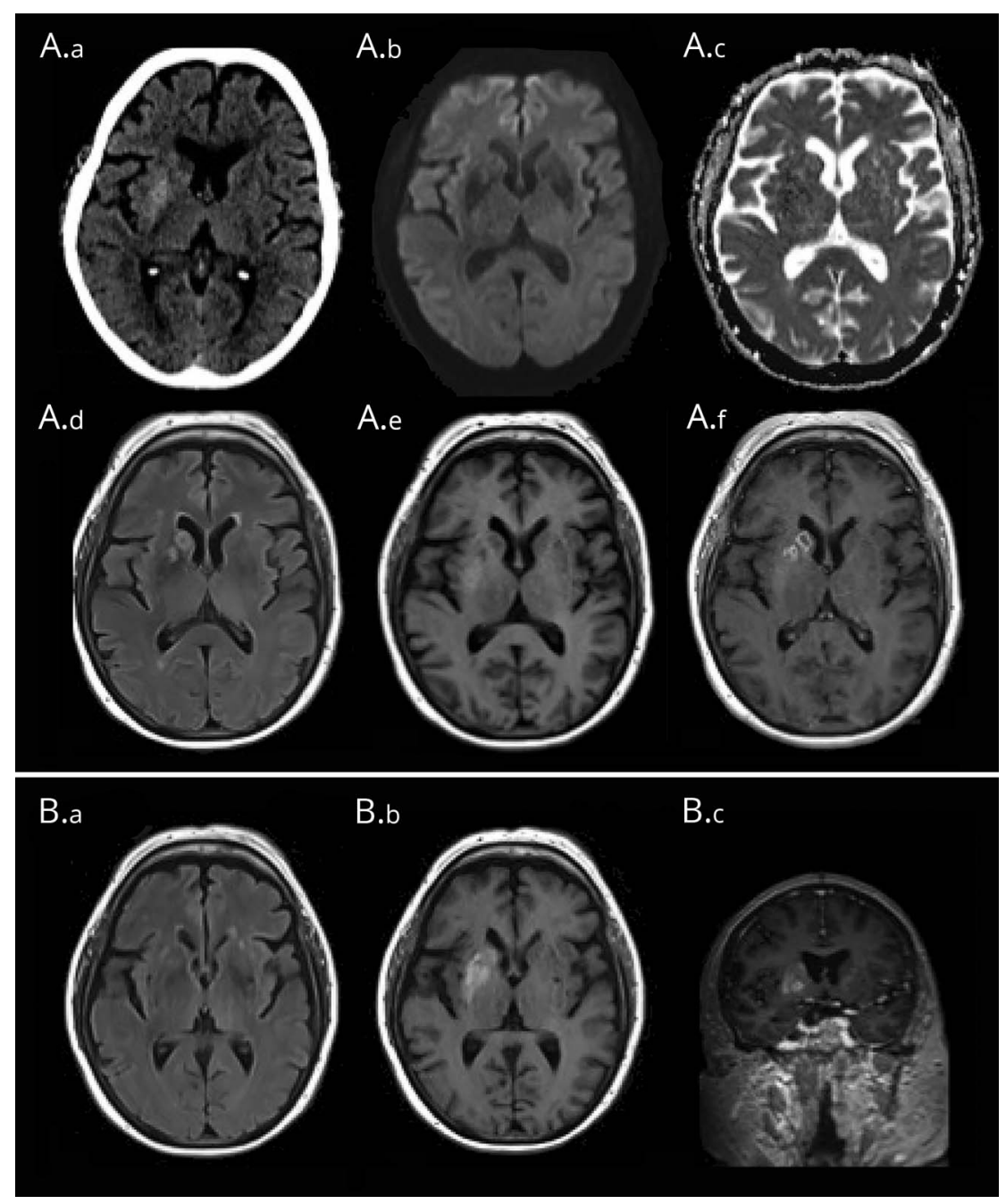

(A) Initial imaging. (A.a) Initial CT: hypoattenuating and subtle expansile appearance of the right caudate head; right putamen subtly hyperattenuating. (A.b-A.c) Initial diffusion: mild increased signal in area of fluid-attenuated inversion recovery (FLAIR) hyperintensity on trace imaging related to increased diffusivity given high apparent diffusion coefficient values. (A.d) Initial FLAIR: rounded areas of increased signal involving caudate head and putamen. (A.e-A.f) Initial T1 pre and post: hypointense appearance of expansile right caudate head and anterior putamen, which shows peripheral enhancement. Subtle increased T1 signal of remainder of putamen. (B) Follow-up imaging at 2 months. (B.a) FLAIR: resolution of hyperintensity involving right lentiform nucleus. (B.b) T1 precontrast: confluent area of T1 shortening involving the right basal ganglia. (B.C) T1 postcontrast: T1 shortening, without enhancement, of the caudate head, putamen, and globus pallidus. Interval right caudate head volume loss. 


\section{Section 3}

The differential for rim/ring-enhancing lesions can be remembered with the mnemonic MAGICAL DR: metastasis, abscess, glioblastoma, infarction (subacute), contusion, aneurysm, lymphoma, demyelination, and radiation necrosis or resolving hematoma. ${ }^{4}$ It may also be worthwhile to consider nonabscess infections such as tuberculomas and neurocysticercosis. The common elements in the differential for acquired causes of hemiballism and ring-enhancing lesions narrow the list to favor infectious, neoplastic, and ischemic etiologies. In addition to the enhancing lesion, imaging was also notable for right putaminal T1 hyperintensity, which suggested a possible metabolic process vs substance deposition of lipid, protein, or hemosiderin. This complicates the differential as none of the favored etiologies should present with this hyperintensity, suggesting a possible secondary pathology or an atypical presentation.

Chart review for background was notable for negative lymph nodes at breast cancer lumpectomy and laboratory studies drawn 8 days prior to presentation that demonstrated a blood glucose level of 599 whereas prior glucose levels were 120-200 with a HbAlc 7.03 months prior to presentation compared to 14 on presentation.

\section{Questions for consideration:}

1. What is your final diagnosis?

2. How would you treat this patient?

GO TO SECTION 4 


\section{Section 4}

The patient presented with subacute onset of left hemiballism 1 month after head trauma in setting of multiple vascular risk factors, acute-on-chronic hyperglycemia, and history of cancer. Imaging was notable for persistent T1 hyperintensity of the right lentiform nucleus and rimenhancing lesions of the right caudate head and anterior putamen progressing to caudate volume loss. She was started on quetiapine for the hemiballism and strict diabetes control was recommended. On follow-up 3 months after symptom onset, her HbAlc was 7.3 and her movements had markedly diminished. She was trialed off of quetiapine but had return of less-severe hemiballism for which she restarted quetiapine. The history, laboratory studies, MRI findings with interval change, and clinical progression indicate that hyperglycemic injury with subacute small vessel infarction is the most likely diagnosis.

\section{Discussion}

In this 65-year-old woman with multiple medical comorbidities, subacute onset of a relatively uncommon movement disorder with atypical imaging findings presented a diagnostic challenge. There is a long differential for acquired causes of hemiballism and of those without more systemic presentations, vascular, metabolic, drug, and structural lesions were thought most likely. While her initial CT was concerning for hemorrhage vs neoplasm, her subsequent MRIs broadened the differential to include infarction, infectious/ inflammatory, and metabolic processes. The ring-enhancing lesion in the basal ganglia was concerning for an infectious etiology such as toxoplasma although she did not fit the classic history. She had no history of being clearly immunocompromised outside of her diabetes, and lacked systemic symptoms or supportive laboratory studies to suggest an infectious etiology. Her symptoms were limited to her left hemibody without encephalopathy, which argued against an autoimmune, inflammatory, or toxic cause, and was further supported by laboratory workup. She had a history of remote breast cancer, which is known to metastasize to the brain, and recent trauma; however, imaging was not consistent with either process. Given the persistent high T1 signal intensity in the putamen, which is characteristic of hyperglycemic injury, ${ }^{5,6}$ and the progressive encephalomalacia of the caudate suggestive of infarction in the clinical setting of acutely elevated glucose near the time of symptom onset with multiple vascular risk factors, the final diagnosis is subacute small vessel infarction with concurrent hyperglycemic injury.

Vascular lesions, either ischemic or hemorrhagic, are the most common cause of nongenetic chorea in the hospital population of patients over 50, with early or delayed hemiballism in $1 \%$ of poststroke patients. ${ }^{7}$ The correlating lesions are in the basal ganglia and adjacent white matter, usually in the territory of the middle cerebral artery or posterior cerebral artery. ${ }^{7}$ Poststroke dyskinesias, of which the most commonly reported is hemichorea-hemiballism, ${ }^{3}$ may present acutely or be delayed by months to years followed by a highly variable course that may be transient, recurrent, persistent, or progressive; however, they are usually self-limited and resolve within 6-12 months. ${ }^{3}$

Hyperglycemic hemiballism is the second most common cause of acute hemiballism. ${ }^{2}$ The first case of hemichoreahemiballism associated with hyperglycemia was described by Bedwell in 1960 and was initially most frequently reported in elderly, typically East Asian, women with diabetes mellitus type $2 .{ }^{5}$ As the hyperglycemia develops, hemiballistic movement emerges, although case reports have suggested both acute as well as delayed dyskinesia appearance. ${ }^{2}$ High signal intensity on T1-weighted images in the putamen is classically associated with this condition. ${ }^{1}$

While diabetes mellitus is a risk factor for dyskinesia due to hyperglycemia, it is also a risk factor for ischemic stroke. Diabetes is independently associated with a $60 \%$ risk for recurrent stroke in those over 65 years old and is a key risk factor for first-ever ischemic stroke. ${ }^{8}$ Therefore, while these distinct etiologies of poststroke hemiballism and hyperglycemiahemiballism are both relatively rare, they both may stem from pathologic hyperglycemia and other poorly controlled vascular risk factors.

In terms of management, strict glycemic control is key for both etiologies as well as secondary stroke prevention. Symptomatically, hemichorea-hemiballism tends to resolve spontaneously, with hyperglycemic hemiballism movements disappearing as soon as hours after glucose correction, although $20 \%$ of patients have persistent hemiballism for longer than 3 months, which is often milder than at presentation. ${ }^{2}$ Stroke-related hemiballism usually resolves within 6-12 months after onset. ${ }^{3}$ However, short-term treatment may be required for symptom control. Pharmacotherapy consists mainly of antidopaminergic therapy with typical or atypical antipsychotics that block the striatal D2 receptors believed to be responsible for dyskinetic activity. ${ }^{3}$ This patient was started on quetiapine and by 3 months had excellent glucose control (HbAlc 7.3) with improved but persistent symptoms (videos 1-3).

\section{Author contributions}

K. Cacic: case write-up and literature review. B. Stephens: case review. F. Cloran: neuroradiology analysis and image selection. A. Frattalone: case review.

\section{Acknowledgment}

The authors thank Grace Balfour, medical videographer, who recorded and edited the associated video footage.

\section{Study funding}

No targeted funding reported. 


\section{Disclosure}

The authors report no disclosures relevant to the manuscript. Go to Neurology.org/N for full disclosures.

\section{References}

1. Bizet J, Cooper CJ, Quansah R, Rodriguez E, Teleb M, Hernandez GT. Chorea, hyperglycemia, basal ganglia syndrome (CF-H-BG) in an uncontrolled diabetic patient with normal glucose levels at presentation. Am J Case Rep 2014;15:143-146.

2. Postuma RB, Lang AE. Hemiballism: revisiting a classic disorder. Lancet Neurol 2003; 2:661-668.

3. Nakawah MO, Lai EC. Post-stroke dyskinesias. Neuropsychiatr Dis Treat 2016;12: 2885-2893.
4. Kang O, Rafaey M. Cerebral ring enhancing lesion (mnemonic). Radiopaedia. Available at: radiopaedia.org/articles/cerebral-ring-enhancing-lesions-mnemonic. Accessed February 23, 2018.

5. Suarez-Vega VM, Almaraz CS, Bernardo AI, Rodriguez-Diaz R, Barrio AD, Gil LM. CT and MRI unilateral brain features secondary to nonketotic hyperglycemia presenting as hemichorea-hemiballism. Case Rep Radiol 2016;2016: 5727138 .

6. Das L, Pal R, Dutta P, Bhansali A. "Diabetic striatopathy” and ketoacidosis: report of two cases and review of literature. Diabetes Res Clin Pract 2017;128:1-5.

7. Ghika-Schmid F, Ghika J, Regli F, Bogousslavsky J. Hyperkinetic movement disorders during and after stroke: the Lausanne Stroke Registry. J Neurol Sci 1997;146: 109-116.

8. Guzik A, Bushnell C. Stroke epidemiology and risk factor management. Continuum 2017;23:15-39.

\section{Subspecialty Alerts by E-mail!}

Customize your online journal experience by signing up for e-mail alerts related to your subspecialty or area of interest. Access this free service by clicking on the "My Alerts" link on the home page. An extensive list of subspecialties, methods, and study design choices will be available for you to choose from-allowing you priority alerts to cutting-edge research in your field!

\section{Neurology ${ }^{\circledR}$ Online CME Program}

Earn CME while reading Neurology. This program is available only to online Neurology subscribers. Read the articles marked CME, go to Neurology.org, and click on CME. This will provide all of the information necessary to get started. The American Academy of Neurology (AAN) is accredited by the Accreditation Council for Continuing Medical Education (ACCME) to sponsor continuing medical education for physicians. Neurology is planned and produced in accordance with the ACCME Essentials. For more information, contact AAN Member Services at 800-879-1960.

\section{Apply for the 2019 Palatucci Advocacy Leadership Program}

The $17^{\text {th }}$ annual Palatucci Advocacy Leadership Forum will place July 18-21, 2019, at the Rancho Bernardo Inn near San Diego, CA. Apply now for this award-winning program to receive training in the areas of grassroots advocacy, leadership, relationship building, and media skills to help you mold your passionate ideas and issues into strategic action plans. The Palatucci Advocacy Leadership Forum will teach you how to:

- Turn your needs and those of your patients into action plans

- Develop media communication skills, whether in front of the camera or through written editorials

- Build and maintain relationships with reporters

- Represent your patients and profession with state and federal representatives

Learn more and apply by the March 4 deadline at AAN.com/view/PALF. 


\section{Neurology}

\section{Clinical Reasoning: A 65-year-old woman with subacute hemiballism \\ Kelsey Cacic, Brian Stephens, Francis Cloran, et al. \\ Neurology 2019;92;345-349 \\ DOI 10.1212/WNL.0000000000006922}

This information is current as of February 11, 2019

Updated Information \& Services

References

Permissions \& Licensing

Reprints including high resolution figures, can be found at: http://n.neurology.org/content/92/7/345.full

This article cites 7 articles, 0 of which you can access for free at: http://n.neurology.org/content/92/7/345.full\#ref-list-1

Information about reproducing this article in parts (figures,tables) or in its entirety can be found online at:

http://www.neurology.org/about/about_the_journal\#permissions

Information about ordering reprints can be found online:

http://n.neurology.org/subscribers/advertise

Neurology ${ }^{\circledR}$ is the official journal of the American Academy of Neurology. Published continuously since 1951, it is now a weekly with 48 issues per year. Copyright (O 2019 American Academy of Neurology. All rights reserved. Print ISSN: 0028-3878. Online ISSN: 1526-632X.

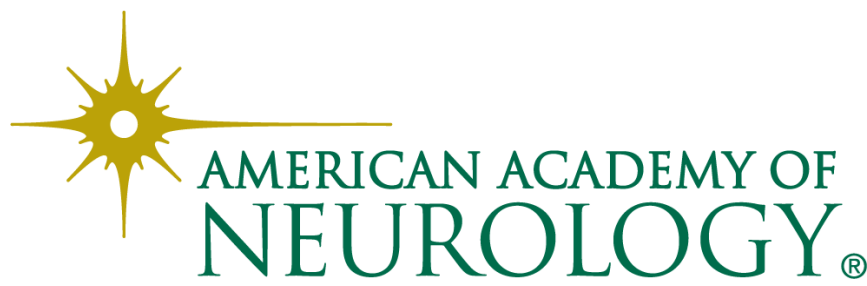

